\title{
SUPPORT PROPERTIES OF GAUSSIAN PROCESSES OVER SCHWARTZ SPACE ${ }^{1}$
}

\author{
M. ANN PIECH
}

ABSTRACT. We utilize the concept of an abstract Wiener space to prove a converse to a theorem of Minlos, thereby obtaining necessary and sufficient conditions for a Hilbert subspace of $S^{\prime}\left(R^{d}\right)$ to support a given Gaussian process over $\delta\left(\mathbf{R}^{d}\right)$.

Stochastic processes over $\mathcal{S}\left(\boldsymbol{R}^{d}\right)$ are of current interest as elements in the construction of relativistic Boson field theories (see Nelson [6], [7]). The basic process corresponding to the free Euclidean field of mass $m$ is the Gaussian process over $\mathcal{S}$ of mean 0 and covariance $\left(g,\left(-\Delta+m^{2}\right)^{-1} f{ }_{L_{L}{ }^{2}\left(\mathbf{R}^{d}\right)}\right.$. According to a theorem of Minlos [4], this process may be realized on $\mathfrak{S}^{\prime}$, the topological dual of $\mathcal{S}$, by $\varphi(f): q \rightarrow\langle f, q\rangle$, where $\langle$,$\rangle denotes the \mathcal{S} \cdot \mathcal{S}$ : pairing. That is, there is a Borel measure $\mu$ on $\mathcal{S}^{\prime}$ so that $\varphi$ maps $\mathfrak{S}$ to Gaussian random variables over $\left(\mathfrak{S}^{\prime}, \mu\right)$ with mean 0 and above specified covariances.

We will say that $\mu$ is supported on a Hilbert space $\mathcal{H}$ if $\mathcal{H} \subset \mathfrak{S}^{\prime}$, the injection of $\mathcal{H}$ into $\mathcal{S}^{\prime}$ is continuous, and there is a Borel measure $\mu_{0}$ on $\mathcal{H}$ so that the restriction of $\varphi(f)$ to $\mathcal{H}$ realizes on $\left(\mathcal{H}, \mu_{0}\right)$ the Gaussian process over $\delta$ of mean 0 and specified covariance. Support properties of $\mu$ have been studied by Reed and Rosen [8]. They utilized a theorem of Minlos to show that certain H's support $\mu$, and they showed by a rather lengthy direct computation that others failed to support. Related results concerning the support of $\mu$ may be found in the recent work of Cannon [1] and of Colella and Lanford [2]. In this note we show that Minlos' sufficient condition for $\mu$ to be supported on $\mathcal{H}$ is, in fact, necessary.

Proposition. Let $(\cdot, \cdot)_{1}$ and $(\cdot, \cdot)_{2}$ be continuous inner products on $\mathfrak{S}$ such that $\|f\|_{2} \leq c\|f\|_{1}$ for some constant $c$ and for all $f$ in $\mathcal{S}$. Let $\mathcal{H}_{1}$ and $\mathcal{H}_{2}$ be the Hilbert space completions of $\mathcal{S}$ with respect to $\|\cdot\|_{1}$ and $\|\cdot\|_{2}$ respectively. Since $\mathcal{S}$ is separable, each $\mathcal{H}_{i}$ is also separable. Let $(\varphi, \mu)$ be the realization on $\mathfrak{S}^{\prime}$ of the Gaussian process over $\mathfrak{S}$ of mean 0 and covariance $(g, f)_{2}$. Then $\mathcal{H}_{1}^{\prime}$ supports $\mu$ if and only if the natural in. jection $\mathrm{H}_{1} \hookrightarrow \mathrm{H}_{2}$ is Hilbert-Schmidt.

Received by the editors August 7, 1974 and, in revised form, October 9, 1974. A.MS (MOS) subject classifications (1970). Primary 60G15; Secondary 81A18; 28 A40. Key words and phrases. Gaussian process, Schwartz space, free Boson field, abstract Wiener space.

${ }^{1}$ Research supported by NSF grant PO28934. 
Remark 1. The following are equivalent definitions of the natural injection $\mathcal{H}_{1} \hookrightarrow \mathcal{H}_{2}$ being $\mathcal{H}_{-\mathcal{S}}$ (Hilbert-Schmidt): (i) (used by Gelfand-Vilenkin [4]) There exists a positive symmetric $\mathcal{H}_{-} \mathcal{S}$ operator $A_{1}$ on $\mathcal{H}_{1}$ such that $(f, g)_{2}=(A f, A g)_{1}$. (ii) (used by Reed-Rosen [8]) There exists a 1-1 $\mathcal{H}-\mathcal{S}$ operator $A_{2}$ on $\mathcal{H}_{2}$ such that $\mathfrak{S} \subset A_{2} \mathcal{H}_{2}$ and $\mathcal{H}_{1}$ is the set $A_{2} \mathcal{H}_{2}$ with norm $\|f\|_{1}=\left\|A_{2}^{-1} f\right\|_{2}$. (iii) There exists a positive symmetric $\mathcal{H}$ - $\mathcal{E}$ operator $A_{3}$ on $\mathcal{H}_{2}^{\prime}$ such that $(q, p)_{1^{\prime}}=\left(A_{3} q, A_{3} p\right)_{2^{\prime}}$.

Remark 2. $\mathcal{S}$ may be replaced by a nuclear space $\mathcal{E}_{\text {. }}$

Proof of Proposition. We make the identifications by injection $\mathfrak{S} \subseteq$ $\mathcal{H}_{1} \subseteq \mathcal{H}_{2}$, by restriction $\mathcal{H}_{2}^{\prime} \subseteq \mathcal{H}_{1}^{\prime} \subseteq \mathcal{S}^{\prime}$ and also canonically identify $\mathfrak{H}_{i}$ with $\mathcal{H}_{i}^{\prime \prime}(i=1,2)$. The map $\varphi(f) q \rightarrow\langle f, q\rangle$ furnishes a densely defined linear mapping of $\mathcal{H}_{1}^{\prime \prime}$ or $\mathcal{H}_{2}^{\prime \prime}$ to Gaussian random variables of mean 0 and covariance specified by the $\mathcal{H}_{2}^{N}$ inner product. Since the $\mathcal{H}_{2}^{\prime \prime}$ inner product is continuous on $\mathcal{H}_{1}^{\prime \prime}$, we obtain a weak distribution [5] over $\mathcal{H}_{1}^{\prime}$ which is the restriction to $\mathcal{H}_{1}^{\prime \prime}$ of the unit normal distribution over $\mathcal{H}_{2}^{\prime}$. This weak distribution uniquely determines a (finitely additive) cylinder set measure $\mu_{1}$ on $\mathcal{H}_{1}^{\prime}$.

$\mathcal{H}_{1}^{\prime}$ supports $\mu$ if and only if $\mu_{1}$ is countably additive on the ring of cylinder sets in $\mathcal{H}_{1}^{\prime}$. The separability of the Hilbert spaces allows us to apply a theorem of Dudley, Feldman and LeCam [3], which asserts that countable additivity of $\mu_{1}$ is equivalent to the pair $\left(\mathcal{H}_{2}^{\prime}, \mathcal{H}_{1}^{\prime}\right)$ forming an abstract Wiener space in the sense of L. Gross [5]. It is well known (and very easy to calculate) that if $\mathcal{K}_{1}$ and $\mathcal{K}_{2}$ are two real separable Hilbert spaces, then $\left(\mathcal{K}_{1}, \mathcal{K}_{2}\right)$ forms an abstract Wiener space if $\mathcal{K}_{2}$ is the completion of $K_{1}$ with respect to an inner product $(f, g)_{2}=(A f, A g)_{1}$, where $A$ is positive $\mathcal{H}-\mathcal{S}$ on $\mathcal{K}_{1}$.

Conversely, we claim that if a real separable Hilbert space $\mathcal{K}_{2}$ arises as the completion of a Hilbert space $K_{1}$ with respect to a continuous norm $\|\cdot\|_{2}$ on $\mathcal{K}_{1}$, and if the pair $\left(\mathcal{K}_{1}, \mathcal{K}_{2}\right)$ forms an abstract $\mathbb{W}$ iener space, then $(f, g)_{2}=(A f, A g)_{1}$ where $A$ is positive, symmetric and $\mathcal{H}_{-} \mathcal{S}$ on $\mathcal{K}_{1}$. Let us make the identifications $\mathcal{K}_{2}^{\prime} \subseteq \mathcal{K}_{1}^{\prime} \approx \mathcal{K}_{1} \subseteq \mathcal{K}_{2}$, the first containment by restriction and the second by the canonical injection. Then it follows from [5, Corollary 5] that the canonical isomorphism $M$ of $\mathcal{K}_{2}$ onto $\mathcal{K}_{2}^{\prime}$ has the property that when restricted to $K_{1}$ and viewed as an operator $M_{1}$ on $K_{1}$, it is positive symmetric and of trace class. But this means that $(f, g)_{2}=$ $\left(f, M_{1} g\right)_{1}=\left(\sqrt{M}_{1} f, \sqrt{M}_{1} g\right)_{1}$, where $\sqrt{M}_{1}$ is $\mathcal{H}-\mathcal{S}$.

\section{REFERENCES}

1. J. Cannon, Continuous sample paths in quantum field theory, Comm. Math. Phys. 35 (1974), 215-233.

2. P. Colella and O. Lanford, Sample field behavior for the free Markov random field, Lecture Notes in Physics, vol. 25, Constructive Quantum Field Theory, Springer-Verlag, Berlin and New York, 1973. 
3. R. M. Dudley, J. Feldman and L. Le Cam, On seminorms and probabilities, and abstract Wiener spaces, Ann. of Math. (2) 93 (1971), 390-408. MR 43 \#4995.

4. I. M. Gel' fand and N. Ja. Vilenkin, Generalized functions. Vol. 4: Some applications of harmonic analysis, Fizmatgiz, Moscow, 1961; English transl., Academic Press, New York, 1964. MR 26 \#4173; 30 \#4152.

5. L. Gross, Abstract Wiener spaces, Proc. Fifth Berkeley Sympos. Math. Statist. and Probability (Berkeley, Calif., 1965/66), vol. II: Contributions to Probability Theory, part I, Univ. California Press, Berkeley, Calif., 1967, pp. 31-42. MR $35 \# 3027$.

6. E. Nelson, Construction of quantum fields from Markoff fields, J. Functional Analysis 12 (1973), 97-112.

7. - Probability theory and Euclidean field theory, Lecture Notes in Physics, vol. 25, Constructive Quantum Field Theory, Springer-Verlag, Berlin and New York, 1973.

8. M. Reed and L. Rosen, Support properties of the free measure for Boson fields, Comm. Math. Phys. 36 (1974), 123-132.

DEPARTMENT OF MATHEMATICS, SUNY AT BUFF ALO, AMHERST, NEW YORK 14226 